\title{
Cosmic ray studies with the Fermi Gamma-ray Space Telescope Large Area Telescope
}

\author{
D.J. Thompson ${ }^{\mathrm{a}, *}$, L. Baldini ${ }^{\mathrm{b}}$, Y. Uchiyama ${ }^{\mathrm{c}}$ \\ a NASA Goddard Space Flight Center, Greenbelt, MD 20771, USA \\ ${ }^{\mathrm{b}}$ Istituto Nazionale di Fisica Nucleare, Sezione di Pisa, I-56127 Pisa, Italy \\ ' SLAC National Accelerator Laboratory, 2575 Sand Hill Road M/S 29, Menlo Park, CA 94025, USA
}

\section{A R T I C L E I N F O}

Article history:

Available online xxxx

\section{Keywords:}

Cosmic rays

Gamma rays

Observations

\begin{abstract}
A B S T R A C T
The Large Area Telescope (LAT) on the Fermi Gamma-ray Space Telescope provides both direct and indirect measurements of galactic cosmic rays (CR). The LAT high-statistics observations of the $7 \mathrm{GeV}-1 \mathrm{TeV}$ electron plus positron spectrum and limits on spatial anisotropy constrain models for this cosmic-ray component. On a galactic scale, the LAT observations indicate that cosmic-ray sources may be more plentiful in the outer Galaxy than expected or that the scale height of the cosmic-ray diffusive halo is larger than conventional models. Production of cosmic rays in supernova remnants (SNR) is supported by the LAT $\gamma$-ray studies of several of these, both young SNR and those interacting with molecular clouds.
\end{abstract}

(c) 2011 Elsevier B.V. All rights reserved.

\section{Introduction}

Among the earliest stimuli for studying cosmic $\gamma$ rays was the recognition by Hayakawa [1] and Morrison [2] that cosmic ray particles interacting with interstellar matter would inevitably produce high-energy $\gamma$ rays. The first high-energy $\gamma$-ray sky map by OSO-3 [3] confirmed this expectation. Gamma-ray and cosmic-ray studies, both observational and theoretical, have been closely linked ever since, with principal results for photon energies above $30 \mathrm{MeV}$ coming from satellite detectors (SAS-2 [4], COS-B [5], EGRET/Compton Gamma Ray Observatory [6]) and ground-based $\mathrm{TeV}$ telescopes (for a recent review, see [7]).

While sharing many experimental techniques, cosmic-ray and $\gamma$-ray studies offer complementary approaches. Cosmic-ray detectors measure the particles directly in the solar neighborhood, including detailed spectral and composition analysis. Observations with $\gamma$-ray telescopes study cosmic-ray interactions at a distance, providing indirect insight about the particles. The $\gamma$-ray observations have the potential to trace sources of cosmic rays, since the photons are not deflected by galactic magnetic fields as the charged particles are.

The present review concentrates on results from the Large Area Telescope on the Fermi Gamma-ray Space Telescope satellite, which was launched on 11 June 2008. The Fermi LAT offers three lines of research related to cosmic rays:

\footnotetext{
* Corresponding author.

E-mail address: David.J.Thompson@nasa.gov (D.J. Thompson).
}

1. Because the LAT is a particle detector, it can make direct measurements of cosmic rays, especially cosmic-ray electrons (CRE).

2. The large-scale $\gamma$-ray properties of our own and other galaxies offer some insight into the distribution of cosmic rays and their sources.

3. The LAT measures the $\gamma$-ray properties of likely acceleration/ interaction sites of cosmic rays such as supernova remnants.

\section{The Fermi large area telescope}

The LAT is a $\gamma$-ray telescope [8], designed to distinguish photons in the energy range $20 \mathrm{MeV}$ to more than $300 \mathrm{GeV}$ from the high background of energetic charged particles at the $565 \mathrm{~km}$ altitude orbit of the Fermi satellite. The tracking section has 36 layers of silicon strip detectors to record the tracks of charged particles, interleaved with 16 layers of tungsten foil ( 12 thin layers, 0.03 radiation length, at the top or Front of the instrument, followed by 4 thick layers, 0.18 radiation length, in the Back section) to promote $\gamma$ ray conversion into electron-positron pairs. Beneath the tracker is a calorimeter comprised of an 8-layer array of CsI crystals (1.08 radiation length per layer) to determine the $\gamma$-ray energy. The tracker is surrounded by segmented charged-particle anticoincidence detectors (ACD; plastic scintillators with photomultiplier tubes) to reject cosmic-ray background events. The LAT's high sensitivity stems from a large peak effective area $\left(\sim 8000 \mathrm{~cm}^{2}\right)$, wide field of view ( $\sim 2.4 \mathrm{sr}$ ), good background rejection, superior angular resolution (68\% containment angle $\sim 0.6^{\circ}$ at $1 \mathrm{GeV}$ for the Front section and about a factor of 2 larger for the Back section), and excellent observing efficiency (keeping the sky in the field of view with 
scanning observations). In scanning mode, the LAT observes the entire sky every 2 orbits (about $3 \mathrm{~h}$ ). Pre-launch predictions of the instrument performance are described in [8].

In addition to being a $\gamma$-ray telescope, the LAT is intrinsically an electron detector. One of the biggest analysis challenges for electron studies is the separation of CRE from the much more abundant hadrons (primarily protons). Key to the background rejection is the LAT capability to discriminate between electromagnetic and hadronic showers, based on the different event topology in the three subsystems. This is largely in common with the photon analysis, the most prominent difference being the use of the ACD.

Unlike previous generations of pair conversion telescopes, the LAT basically triggers on all the charged particles crossing the active volume, with no harsh photon selection built into the hardware trigger. This drastic change of approach is largely due to the use of silicon detectors, allowing precise tracking with essentially no detector-induced dead time. Some event filtering is necessary prior to downlink in order to fit the data volume into the allocated telemetry bandwidth. The onboard filter is really tailored to preserve the $\gamma$-ray efficiency by discarding events that are unlikely to be $\gamma$ rays. The rate of events depositing more than $20 \mathrm{GeV}$ into the calorimeter, however, is small enough that we can afford effectively to disengage the filter above that energy, transmitting to the ground high-energy charged particle events along with the $\gamma$ rays.

\section{Direct cosmic ray measurements}

A century after the pioneering cosmic-ray studies by Domenico Pacini and Victor Francis Hess we have a huge body of knowledge about these energetic particles. In the LAT energy range, many instruments have measured the energy spectra of CR particles and their composition, including not only protons and electrons, but also heavier nuclei. They can be roughly divided into inclusive measurements (i.e. $p$ and $e^{-}+e^{+}$spectra) and measurements of the charge composition (i.e. $\bar{p} /(p+\bar{p})$ and $\left.e^{+} /\left(e^{-}+e^{+}\right)\right)$, providing complementary information on the origin, acceleration and propagation of cosmic rays. This dichotomy has a parallel in the variety of experimental techniques routinely exploited since the mid1960 's, in that cosmic-ray instruments generally fall in one of the two categories: calorimetric experiments (that cannot distinguish, per se, the charge sign) and magnetic spectrometers.

Fig. 1 is a partial compilation of such instruments, classified by acceptance $^{1}$ and effective observation time. It is not a trivial task to give an exhaustive and unbiased account of the huge experimental effort that turned the first observations into precision science. In many cases the geometry factor is energy dependent, and some modern instruments (particularly the magnetic spectrometers) can operate in different configurations with different acceptances. In addition, each detector is sensitive in a different energy band (for any given observable), which makes a fair comparison extremely difficult. Despite these caveats, Fig. 1 is accurate enough to illustrate some interesting aspects of the historical development of cosmicray measurements: calorimetric experiments feature, in general, a larger acceptance than magnetic spectrometers; and space experiments have the obvious advantage of a relatively longer collection time. Fig. 1 also puts Fermi into the context of this development: the LAT's unique combination of large acceptance and long observation time results in an unrivaled exposure factor ${ }^{2}$ exceeding $10^{8}$ $\mathrm{m}^{2} \mathrm{sr} \mathrm{s}$, as indicated by the diagonal gray lines.

\footnotetext{
1 The acceptance, or geometry factor $\left(G_{\mathrm{F}}\right)$, is the integral of the effective area over the instrument field of view and constitutes the proportionality factor between the event rate in the detector and the incident flux.

2 The exposure factor is the product of the geometry factor and the effective observation time and is directly related to the total number of events recorded by a given instrument.
}

\section{Direct CR measurements with Fermi}

High-energy cosmic-ray electrons and positrons constitute a peculiar component of the cosmic radiation in that, unlike protons and heavier nuclei, they rapidly lose energy by synchrotron radiation on galactic magnetic fields and by inverse Compton (IC) scattering on the interstellar radiation field. In the energy range of interest for the LAT-i.e. above a few $\mathrm{GeV}$, where losses dominate over escape-the average lifetime for electrons against synchrotron and IC losses is $\tau_{e}(E) \propto E^{-1}$, whereas the corresponding escape time for protons scales with the inverse of the diffusion coefficient: $\tau_{p}(E) \propto E^{-\delta}$ (with $\delta \approx 0.3-0.6$ ). This implies that the all-electron spectrum at Earth is significantly steeper than the proton spectrum. More importantly, at energies above a few hundred $\mathrm{GeV}$ the majority of the electron and positron flux must be contributed by sources closer than a few hundred pc. In other words, high-energy CRE really probe cosmic-ray production and propagation in nearby or local galactic space.

The other piece of conventional wisdom is that unlike electrons, which are accelerated in CR sources, the vast majority of positrons arise as secondary products of cosmic-ray interactions in our Galaxy. In the absence of primary sources of positrons it is straightforward to demonstrate that in the simplest scenarios the positron fraction is expected to decrease with energy like $E^{-\delta}$, e.g. [9]. As we shall see in a moment, this expectation is at odds with the most recent measurements [10].

Fermi began its operations at about the same time two important measurements $[11,10]$ were published that challenge the simplest models of cosmic-ray production and propagation. These reports stirred an enormous excitement in the community, mainly in connection with the possibility of detecting signs of new physics in the context of indirect dark matter searches. In 2009 the Fermi LAT collaboration published the first systematics-limited measurement of the inclusive spectrum of cosmic-ray electrons and positrons [12] between $20 \mathrm{GeV}$ and $1 \mathrm{TeV}$. Though harder than previously thought, the Fermi spectrum does not show evidence of any prominent feature, such as the one reported by the ATIC experiment [11]. This conclusion was later independently confirmed by the H.E.S.S. [13] and Pamela [14] experiments, as well as by a dedicated analysis of the Fermi data aimed at the highest end of the energy range [15]. Fig. 2 summarizes the current status of measurements.

The Fermi measurement-especially when the low-energy extension of the spectrum published in [15] is taken into account-is in tension with a single power-law model, though this cannot be definitely ruled out within the systematic uncertainties. This tension has motivated a number of papers in which a consistent interpretation of the Fermi inclusive spectrum and the rise in the positron fraction above $\approx 10 \mathrm{GeV}$ measured by Pamela [10] is attempted through the introduction of an additional nearby source of high-energy electrons and positrons (for a more detailed discussion see [17] and references therein). It is fair to say that, to date, the issue is not settled, and there is no firm consensus on whether observations really indicate the existence or nature of this extra component. Indeed there are alternative scenarios (see for instance $[18,19]$ ) that might explain the observations without invoking any new (astrophysical or exotic) source of high-energy electrons and positrons.

For the future, with more than 5000 electrons above $1 \mathrm{TeV}$ per year crossing the LAT, Fermi can hope to provide an independent confirmation of the spectral cutoff measured by H.E.S.S. [20] in this energy range. Even with a selection efficiency on the order of $10 \%$ (in line with the figures of merit of the current analysis), the statistics will not be an issue. At the same time, the collaboration is actively working on the energy measurement, which is the most challenging part of the analysis. 


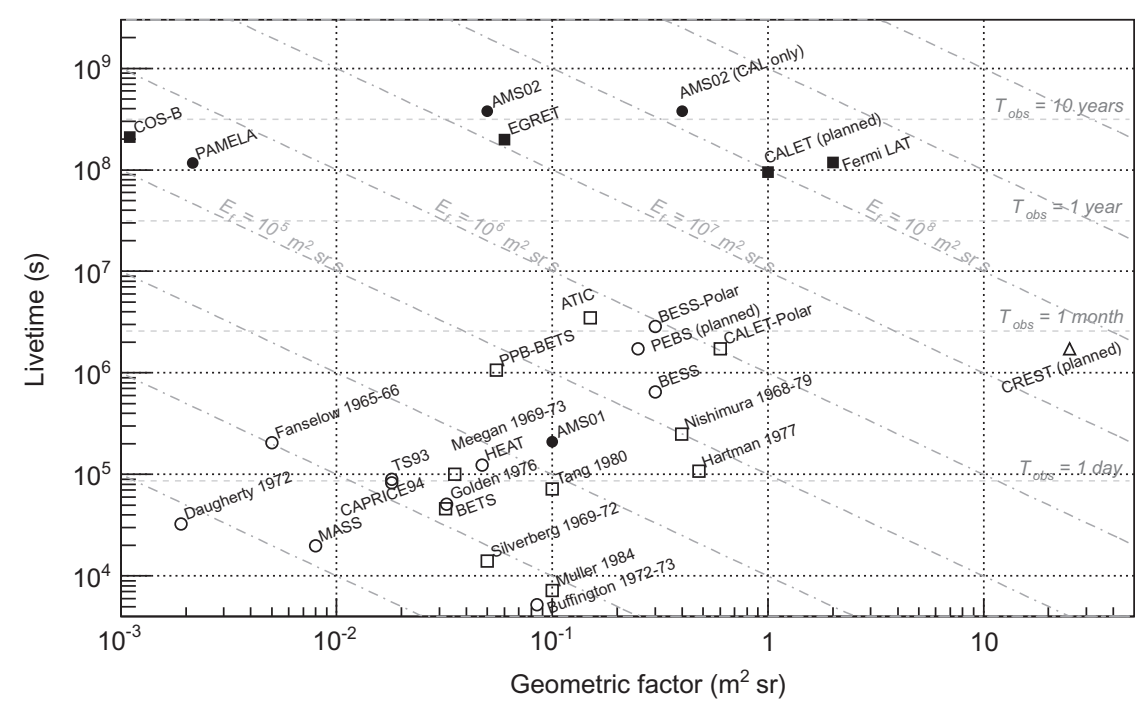

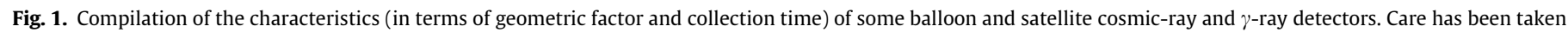

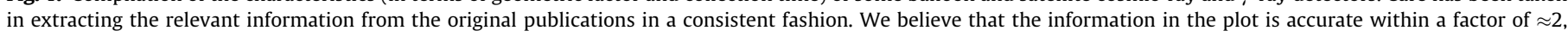

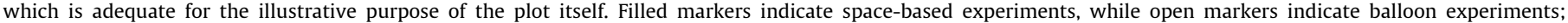
conversely squares indicate calorimetric experiments, while circles indicate magnetic spectrometers.

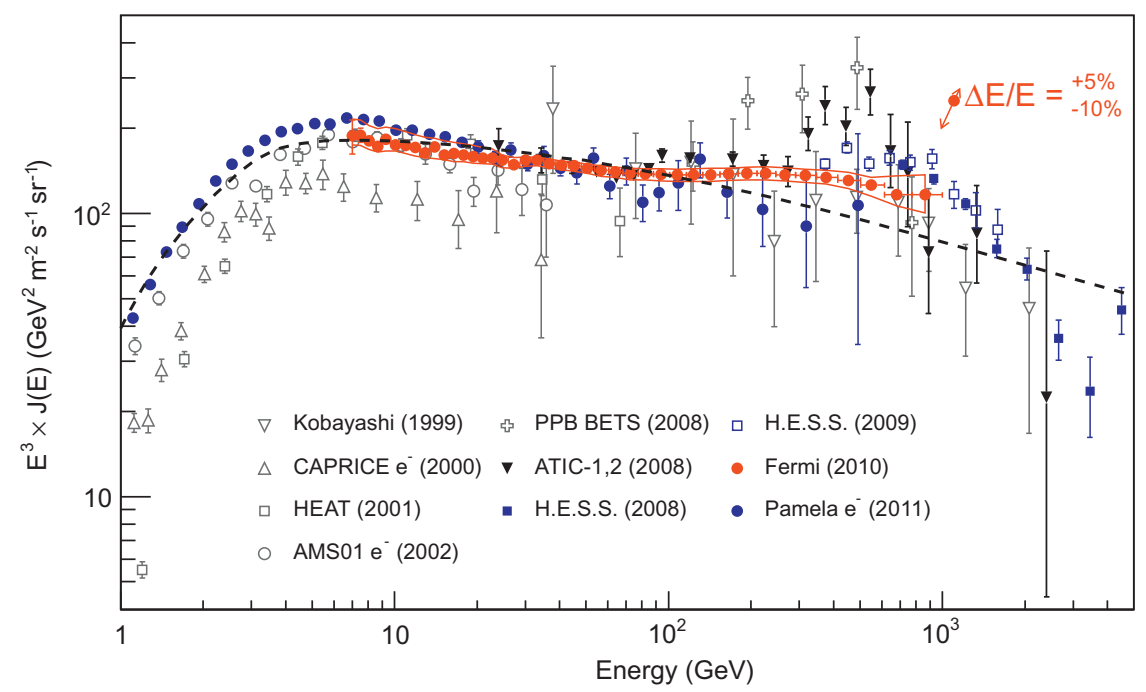

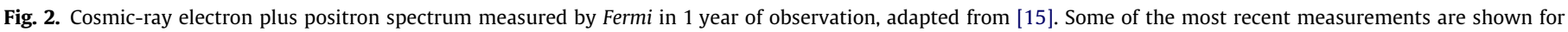

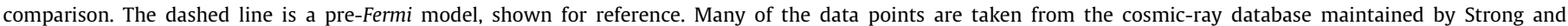
Moskalenko [16].

Without an onboard magnet, Fermi LAT cannot directly distinguish the charge sign. However the Earth's magnetic field creates natural shadow regions from which particular charges are forbidden-simply because their paths are blocked by the Earth-and can be effectively exploited in order to separate positrons from electrons. The idea of using the geomagnetic field to study the charge asymmetry in the leptonic component of cosmic rays is not new. In fact the possibility of using the east-west asymmetry in the geomagnetic cutoff rigidity has been explored in several balloon experiments (see [21] and references therein for more information). In addition to its large field of view and its excellent directional capabilities, Fermi can take advantage of the accurate models of the Earth's magnetic field [22] and the particle tracing computer programs that are nowadays readily available. One of the main challenges in the analysis is the fact that, as the energy increases, the shadow regions become smaller and smaller. Coupled with the falling spectrum, this effect in practice limits the highest accessible energy to $\approx 200 \mathrm{GeV}$. In addition to that, these regions are located near the Earth's horizon, which is outside or at the limits of the LAT field of view in the nominal sky-survey operations. Preliminary results of this analysis are in agreement with the positron fraction measured by Pamela and will be the subject of a forthcoming paper. With the AMS detector now installed on the International Space Station, new data in the unexplored high-energy range will be available in the near future.

The electron component of cosmic rays is not fully described by the inclusive energy spectrum and the positron fraction: the degree of anisotropy in the arrival directions is an additional key observable, especially in a scenario in which the inclusive electron spectrum is dominated by one (or a few) nearby sources. Some degree of anisotropy is indeed expected even in a plain diffusive scenario in which the sources are continuously distributed, just due to the fact that the Earth is not at the center of the diffusion halo. It is natural to expect that the flux anisotropy can be much larger if a 


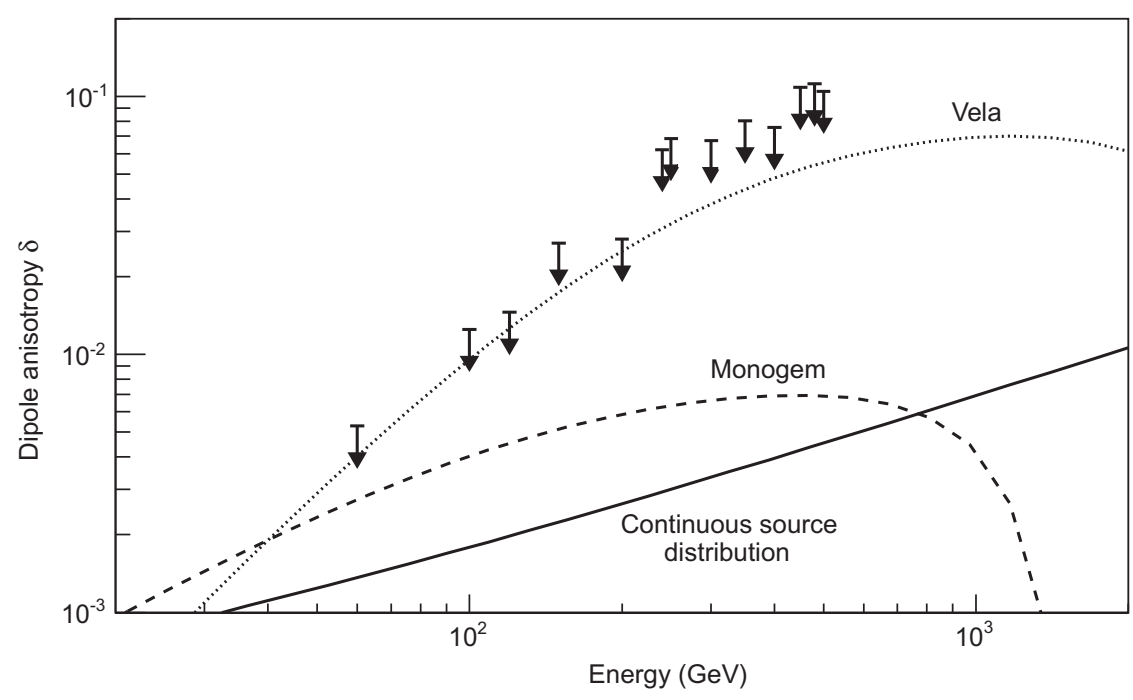

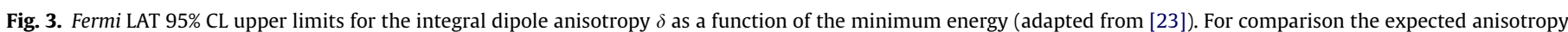

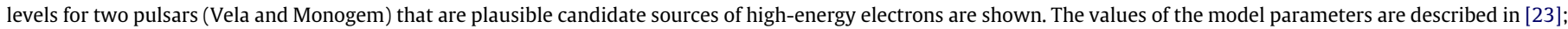

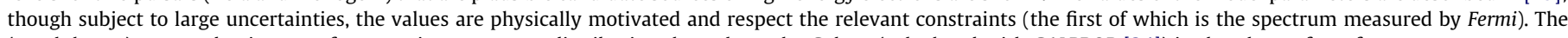
(much lower) expected anisotropy for a continuous source distribution throughout the Galaxy (calculated with GALPROP [24]) is also shown for reference.

single (or a few) nearby sources dominate the CRE spectrum at high energy.

Key to these measurements are Fermi's large geometric factor, its excellent directional capabilities and its characteristic of an all-sky monitor providing uniform exposure. ${ }^{3}$

Fermi has published the most stringent upper limits to the anisotropy of the CRE flux, based on the first year of data [23]. The paper discusses all aspects of the analysis: the pixelization of the sky, the pixel-to-pixel fluctuations, the search for anisotropies on different angular scales (from $10^{\circ}$ to $90^{\circ}$ ) and a full spherical harmonic analysis. In this review we concentrate on a single aspect, namely the upper limits on a possible dipole anisotropy, which is physically relevant for a single source dominating the spectrum (in which case the source defines one of the poles of the dipole).

Some of the basic results are shown in Fig. 3. Though entering a detailed discussion of specific models and related parameter space is beyond the scope of this review, we emphasize that Fermi is already starting to constrain physically motivated scenarios and might hope to detect a positive signal during the lifetime of the mission (5-10 years). If indeed there is a nearby source of high-energy electron/positron pairs, this signal would be a powerful tool to shed light on its nature. For most of the dark matter scenarios one would expect an excess toward the galactic center (potentially at the same level as the ones shown in the figure) while Monogem and Geminga, which are two good pulsar candidates, are roughly in the opposite direction.

\section{Diffuse galactic gamma rays as probes of galactic cosmic rays}

Unlike the sky at visible wavelengths, the $\gamma$-ray sky is strongly dominated by diffuse radiation originating in our Milky Way Galaxy, making the galactic plane the most striking feature in any large-scale $\gamma$-ray skymap. Although the diffuse galactic $\gamma$ radiation is brightest along the plane, it is seen at all galactic latitudes. This radiation is largely produced by cosmic-ray interactions with the interstellar gas and photon fields through the processes of inelastic

\footnotetext{
${ }^{3}$ The exposure is not actually uniform over the sky; there is a non-uniformity at the level of $15 \%$, mainly due to the fact that the instrument is not taking data while inside the South Atlantic Anomaly. This is described in [23].
}

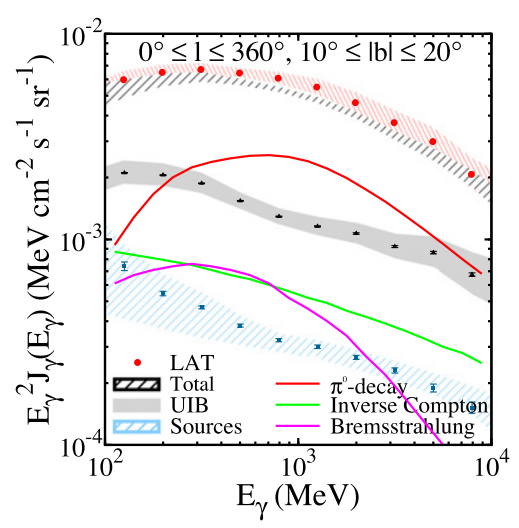

Fig. 4. Spectral energy distribution (SED) of diffuse $\gamma$ radiation at intermediate galactic latitudes. Overlaid are SEDs for the component processes of the diffuse emission. The UIB component is unidentified isotropic background, including instrumental background. The primary component, shown by the heavier line above the gray band, is decay of $\pi^{0}$ mesons [25].

nucleon scattering, bremsstrahlung, and inverse Compton scattering. Fig. 4 shows the Spectral Energy Distribution (SED) of this radiation in part of the sky, along with modeled components [25]. The largest contributor to this radiation is nucleon-nucleon collisions (basically cosmic ray protons and heavier nuclei hitting interstellar hydrogen nuclei) with subsequent decay of $\pi^{0}$ mesons into $\gamma$ rays in the Fermi LAT energy range. The LAT measurements do not confirm the unexpected excess in the few $\mathrm{GeV}$ energy range measured by EGRET on the Compton Gamma Ray Observatory compared to models.

Mapping the galactic $\gamma$ rays offers a way to derive information about the spatial distribution of cosmic-ray particles. The modeling necessary to extract such results is not simple because it requires knowledge of all the matter and photon distributions with which the cosmic rays interact. Models for distribution of cosmic-ray sources and propagation include GALPROP [26] ${ }^{4}$ and a derivative, DRAGON [27]. Fig. 5 shows one result that has been derived from the LAT results: an indication that conventional models for CR source

\footnotetext{
${ }^{4}$ see also http://galprop.stanford.edu/
} 


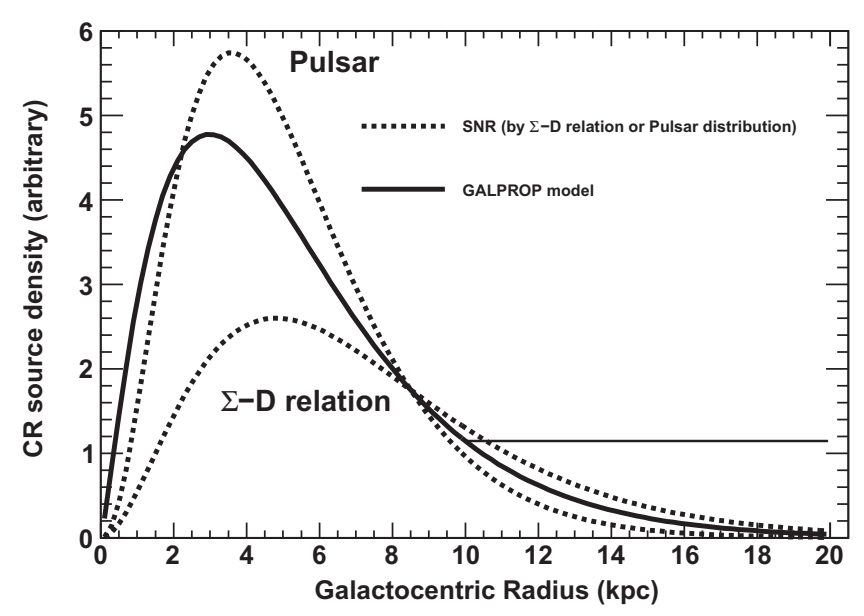

Fig. 5. CR source distribution from one GALPROP model (solid line), compared with the SNR distribution obtained by the $\Sigma$-D relation [31] (see [32] for a critique of this method, however) and that traced by the pulsar distribution [33] shown by dotted lines. The thin solid line represents an example of the modified distributions introduced to reproduce the emissivity gradient observed by the LAT [28].

distributions underpredict the observations in the outer Galaxy. As noted in [28], the observed $\gamma$-ray emissivity gradient is inconsistent with all available tracers of massive star formation. In this case the LAT results suggest either a large scale height for the CR diffusive halo or a flatter distribution of the sources beyond the Solar circle, although other possibilities cannot be ruled out, including a large mass of unseen gas in the outer Galaxy [29], a non-uniform diffusion coefficient [27], or advective CR transport by a galactic wind [30].

Some galaxies not exhibiting apparent nuclear jet activities are also seen as $\gamma$-ray sources that offer information about cosmic rays beyond our own Galaxy. Examples are normal galaxies, including the Large Magellanic Cloud [35], the Small Magellanic Cloud [34], and starburst galaxies, such as M82 and NGC 253 [36]. GeV $\gamma$ rays in these galaxies come primarily from the interactions of cosmic ray hadrons and electrons with interstellar matter and photon fields. Fig. 6 shows the measured spectrum from the Small Magellanic Cloud. The spectrum is modeled predominantly by $\pi^{0}$ decay, implying hadronic processes similar to those in our own Galaxy. The detailed analysis of the LMC shows that the CR flux there is less

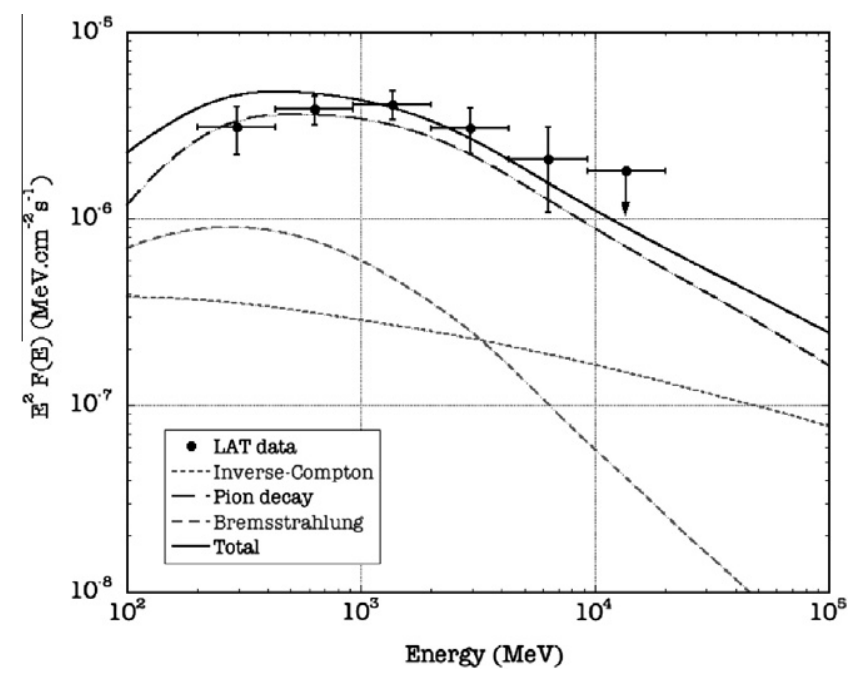

Fig. 6. SED of $\gamma$ radiation from the small magellanic cloud. Overlaid are SEDs for the component processes of the diffuse emission. The primary component is decay of $\pi^{0}$ mesons [34]. than a third that of the Milky Way [35], and the derived CR flux in the SMC is even lower [34]. The $\gamma$-ray luminosities of these normal and starburst galaxies show an approximately linear relationship with the product of the supernova rate and the total mass of gas in the galaxies [36]. The suggestion is that the $\gamma$-ray production requires both target material (gas) and CR production (traced by the supernova rate). Although this analysis is highly simplified, the trend supports the expectation that the principal accelerators of cosmic rays are in some way related to massive-star-formation activity.

\section{Supernova remnants and other candidate cosmic-ray sources}

\subsection{SNRs as cosmic-ray sources}

Supernova remnants (SNRs) have long been thought to be the main sources of galactic CRs [37], accelerating CR particles at their expanding shock waves through the diffusive shock acceleration (DSA) process [38]. In this standard paradigm, SNRs are responsible for the production of cosmic-ray protons and nuclei up to energies of $\sim 3 \times 10^{15} \mathrm{eV}$, transferring $\sim 10 \%$ of the explosion kinetic energy into the form of cosmic-ray energy [39]. The observations of $\gamma$ rays from SNRs have been viewed as the most promising method of addressing the SNR paradigm for the origin of cosmic rays, through the measurements of the $\pi^{0}$-decay (or hadronic) $\gamma$ rays [40]. Despite the substantial progress of $\mathrm{TeV} \gamma$-ray observations in recent years [7], finding a convincing case of $\pi^{0}$-decay $\gamma$ rays remained tantalizingly difficult, mainly because electromagnetic radiation processes involving relativistic electrons (the so-called leptonic components) could explain the $\gamma$-ray emission as well. It has widely been expected that the Fermi LAT could identify the hadronic component of the $\gamma$-ray emission in SNRs. Indeed the observations with the Fermi LAT have started to provide an effective means to disentangle the leptonic (IC scattering and relativistic bremsstrahlung) and hadronic ( $\pi^{0}$-decay) $\gamma$-ray components.

Gamma-ray observations with the Fermi LAT have so far resulted in the detections of $\mathrm{GeV} \gamma$-ray emission from more than ten SNRs [41-48]. Fig. 7 summarizes the observed GeV luminosity

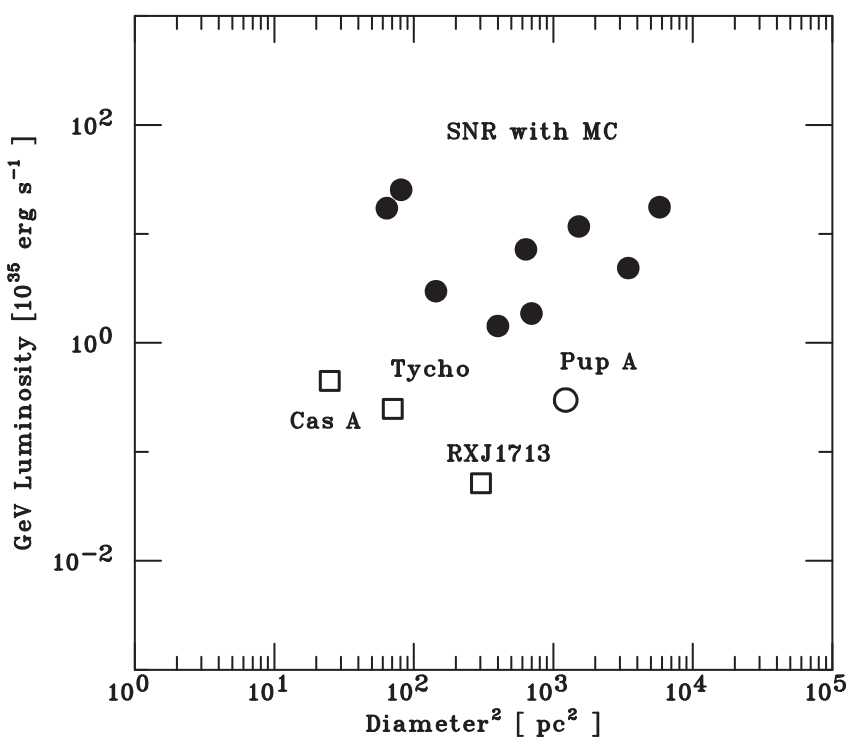

Fig. 7. GeV luminosity $(0.1-100 \mathrm{GeV})$ in units of $10^{35} \mathrm{erg} \mathrm{s}^{-1}$ plotted as a function of diameter squared for the SNRs detected with the Fermi LAT. Filled circles correspond to the SNRs interacting with molecular clouds. Rectangles are young SNRs. Pup A, middle-aged without clear molecular interactions, is shown as an open circle. 
in $0.1-100 \mathrm{GeV}$ as a function of the diameter squared for the known LAT-detected SNRs. Note that the squared diameter would be regarded as a reasonable indicator of SNR ages, as the age of a remnant in the Sedov-Taylor phase is related to its radius as $t \propto R^{2.5}$. The remnants interacting with molecular clouds are indicated by filled circles. They constitute the dominant class of the LAT-detected SNRs, and they are generally more luminous in the $\mathrm{GeV}$ band than young SNRs [49]. The observed $\gamma$-ray luminosity of the SNRs interacting with molecular clouds is so high that one of the main leptonic radiation channels, namely IC scattering off the cosmic microwave background (CMB) and interstellar radiation fields, becomes unlikely. For an efficiency of $\sim 10 \%$ for CR proton production and an electron-to-proton ratio of 0.01 , the GeV luminosity of the IC emission is calculated to be $L_{\mathrm{IC}} \sim 10^{34} \mathrm{erg} \mathrm{s}^{-1}$ using the interstellar radiation fields of the solar neighborhood. The observed luminosity well exceeds this value as shown in Fig. 7. In what follows, we review the results obtained with the LAT in the context of the SNR paradigm for CR origin.

\subsection{Young supernova remnants}

The remnants of historical supernovae are generally well studied across the electromagnetic spectrum, making them excellent laboratories for studying high-energy phenomena associated with supernova shocks, and for testing our current understanding of the DSA theory. The following issues can be addressed by the Fermi LAT observations of young SNRs:

(i) Acceleration efficiency-About $10 \%$ of the mechanical energy of supernova explosions must be transferred to the kinetic energy of CR protons in order to maintain the density of galactic CRs. Since the detailed mechanisms of how thermal particles are injected into the acceleration process and therefore the efficiency of particle injection remain uncertain, the current theory does not firmly predict the amount of CR protons in supernova shocks. The amount of CR electrons produced at shocks is even more uncertain theoretically. (ii) Magnetic field amplification- The DSA process at supernova shocks depends strongly on the generation of magnetic field turbulence by $C R$ themselves ahead of the shock wave. It has been predicted that the turbulent magnetic field in the strong shocks of young SNRs can be significantly amplified by CR current-driven instability [50] and other instabilities. Evidence for the magnetic field amplification was provided by high angular resolution observations with the Chandra X-ray Observatory. Narrow widths of synchrotron X-ray filaments [51,52] and their short time variability [53] require strong magnetic fields. Acceleration of protons up to the knee in the CR spectrum at $\sim 10^{15} \mathrm{eV}$ can be realized only if magnetic field amplification occurs. Gamma-ray observations can put an important constraint on the strength of magnetic field, since the $\gamma$-ray flux due to IC scattering is scaled simply by magnetic field strength given the observed flux of synchrotron radio emission.

(iii) Acceleration spectrum - In the test-particle regime, the DSA theory predicts the spectrum of CRs accelerated at SNR shocks obeying a power law $Q(E) \propto E^{-s}$ with $s \simeq 2$. The CR spectra measured directly at Earth have the form $N(E) \propto E^{-2.7}$ below the knee energy, as confirmed by the Fermi LAT measurements of the galactic diffuse $\gamma$-ray emission. Then the diffusion coefficient for $\mathrm{CR}$ propagation should be roughly $D(E) \propto Q(E) /$ $N(E) \propto E^{0.7}$. Such a strong energy dependence would be inconsistent with the CR anisotropy measurements [54,55]. Recently it has been suggested that the finite velocity of scattering centers (usually neglected in the DSA theory) due to magnetic field amplification may lead to steeper spectra of accelerated CRs with $s \sim 2.2$ [56], though the prediction depends on poorly understood properties of the magnetic turbulences produced at shocks. In this regard, measurements of the $\mathrm{GeV} \gamma$-ray spectrum, especially that of hadronic origin, are of great importance.

\subsubsection{Cas $A$}

Cas A is thought to be the remnant of a Type IIb supernova that exploded around AD 1680. Since it is at the early Sedov-Taylor phase, the accelerated $C R$ spectrum can reach already its maximum

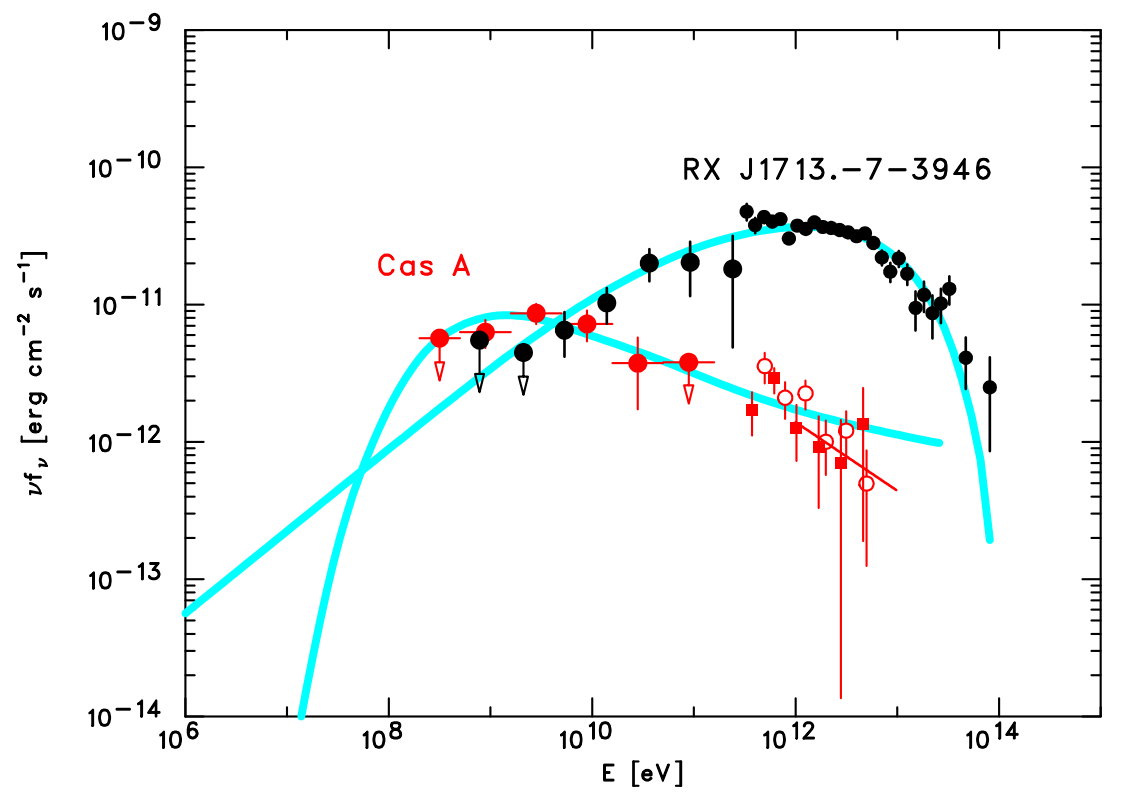

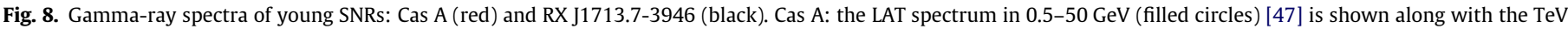

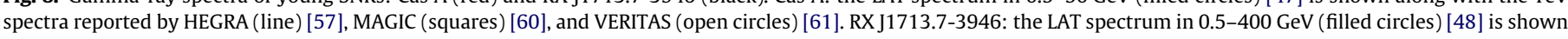

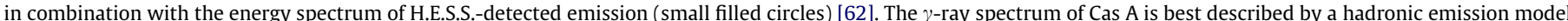

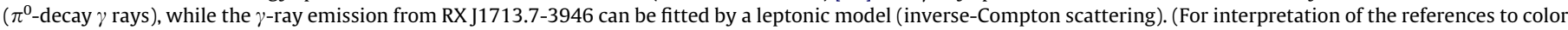
in this figure legend, the reader is referred to the web version of this article.) 
attainable energy and the process of energy conversion, from the expansion kinetic energy into CR energy, can well proceed. Cas A was the first SNR detected in the TeV $\gamma$-ray band [57]. Though the emission mechanisms responsible for the $\mathrm{TeV} \gamma$ rays remained unsettled, their detections provided direct evidence for particle acceleration beyond $10 \mathrm{TeV}$. Observations with EGRET resulted in setting upper limits on the $\gamma$-ray flux above $100 \mathrm{MeV}$.

A clear detection of Cas A at a significance level of $12 \sigma$ was made with the Fermi LAT using the $\gamma$-ray data during the period between 2008 August 4 and 2009 September 4 [47]. The GeV $\gamma$-ray data were consistent with a point-like source, as expected from the angular size of the remnant (a radius of $2.5^{\prime}$ ), and also consistent with steady emission. The $\gamma$-ray spectrum measured for Cas $\mathrm{A}$ in the GeV-TeV band is shown in Fig. 8 .

The X-ray observations of synchrotron-emitting filaments measure the magnetic field at the forward shock; $B \simeq 0.3 \mathrm{mG}$ is inferred from the observed width of the filaments [58], which is consistent with the variability timescale of the synchrotron filaments [59]. By combining the estimated magnetic field with the synchrotron spectrum in the radio bands, it was shown that the leptonic $\gamma$ ray emission model does not well explain the LAT-detected emission [47]. Instead, the GeV-TeV $\gamma$-ray emission can be ascribed mainly to the $\pi^{0}$-decay component. The total proton content at the current age of the remnant amounts to $W_{p} \simeq 0.4 \times 10^{50} \mathrm{erg}$ for the shocked gas density of $n_{\mathrm{H}}=10 \mathrm{~cm}^{-3}$, which is estimated from the measured dynamics of the remnant. This would be the first case where one can estimate the amount of CRs in SNRs with a reasonable accuracy. The spectral slope of accelerated CR protons is assumed to be $s=2.3$ from the radio spectral index (Fig. 8), which seems compatible with the $\mathrm{GeV}-\mathrm{TeV}$ spectrum. Both the CR content and spectrum are in reasonable agreement with the direct measurements of the CR flux and the CR anisotropy.

\subsubsection{Tycho}

Tycho's SNR is the remnant of a Type Ia explosion in 1572, currently in transition from the ejacta-dominated phase to the Sedov-Taylor phase. Recent detection of $\mathrm{TeV} \gamma$-rays from Tycho's SNR by the VERITAS Collaboration [63] has just been followed by the very recent $5 \sigma$ detection of $\mathrm{GeV} \gamma$-ray emission with the Fermi LAT [64]. The LAT spectrum is characterized by photon index $\Gamma=2.3 \pm 0.2$ (stat) \pm 0.1 (sys), which smoothly connects with the VERITAS spectrum.

Given the amplified magnetic field of $0.2-0.3 \mathrm{mG}$ estimated for shock downstream [65,58] and the constraint on the ambient density $\left(n_{\mathrm{H}}<0.3 \mathrm{~cm}^{-3}\right)$, the $\gamma$-ray spectrum of Tycho's SNR can be explained only by the $\pi^{0}$-decay emission. Compared with Cas A, the ambient density is smaller and the radio emission is much weaker, so that the leptonic scenario faces a more serious difficulty. The total CR proton content at the current age amounts to $W_{p} \simeq(0.6-1.5) \times 10^{50}$ erg for a reasonable range of SNR parameters such as distance. The accelerated CR protons and electrons with number index $s=2.3$ are inferred from the multiwavelength fitting. Such a steep spectrum may be explained by the finite velocity of the scattering waves in the shock upstream region [66]. As for Cas A, the LAT results obtained for Tycho's SNR strengthen the case for SNR origin of the galactic CRs.

\subsection{3. $R X J 1713.7-3946$}

SNR RX J1713.7-3946 (G347.3-0.5 in the Green's SNR list [67]) is well known for its bright synchrotron X-ray emission that completely dominates the radiation output from the remnant $[68,69]$ and also for its bright $\mathrm{TeV} \gamma$-ray emission that well traces the synchrotron X-ray map [70,62]. Since its discovery, the origin of the TeV $\gamma$-ray emission has attracted strong interest from the high-energy astrophysics community [71,72].

Fig. 9 presents the Test Statistic (TS) map of a sky region around SNR RX J1713.7-3946 calculated using the events above $0.5 \mathrm{GeV}$ taken from 2008 August 4 to 2010 August 4 [48]. (TS is defined as TS $=-2 \ln \left(L_{0} / L_{\text {point }}\right)$ where $L_{0}$ is the likelihood value of the null hypothesis that takes account of only background sources in the model, and $L_{\text {point }}$ is that of the hypothesis assuming a point source at each location.) The extended $\gamma$-ray emission coincident with SNR RX J1713.7-3946 can be seen in Fig. 9, though it was hard to investigate internal spatial structures due to the lack of photons.

In Fig. 8, the energy spectrum of SNR RX J1713.7-3946 measured with the Fermi LAT [48] is shown together with that of the H.E.S.S. telescopes [62]. The LAT spectrum can be characterized by a hard power law with photon index $\Gamma=1.5 \pm 0.1$ (stat) \pm 0.1 (sys), smoothly connecting with the steeper $\mathrm{TeV}$ spectrum. The hard power-law shape agrees with the expected IC spectrum (the leptonic model), as illustrated in Fig. 8. Given the energy flux ratio of the observed synchrotron X-ray emission and the $\gamma$-ray emission, the leptonic model requires that the average magnetic field be weak, $B \simeq 10 \mu \mathrm{G}$ [62]. If the leptonic scenario is the case, the filamentary structures and variability in X-rays [53] would be attributable to locally enhanced magnetic fields, e.g., time-variable local turbulent magnetic fields [73]. Importantly, within the leptonic scenario the $\gamma$-ray spectrum provides a robust estimate of the total amount of relativistic electrons, as $W_{e} \sim 1 \times 10^{48} \mathrm{erg}$.
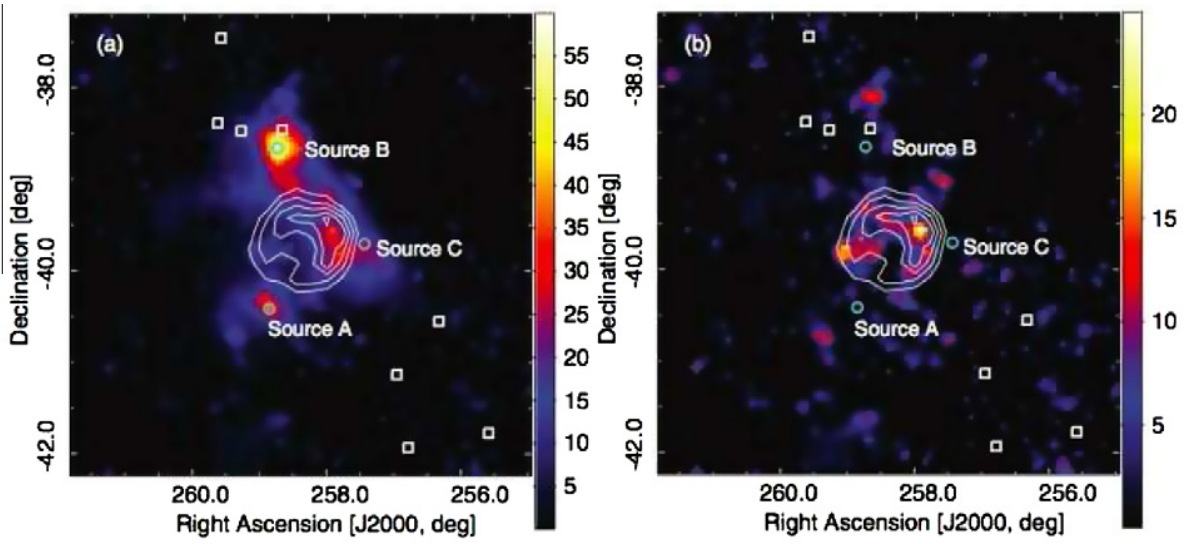

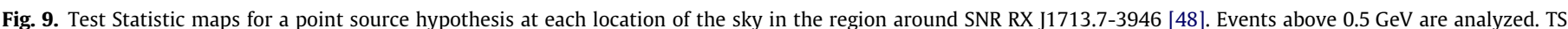

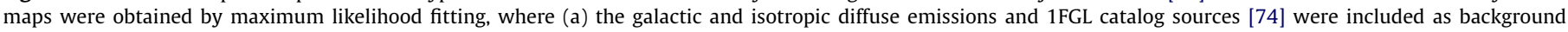
sources, (b) sources A, B, and C were also taken into account in the background model. 

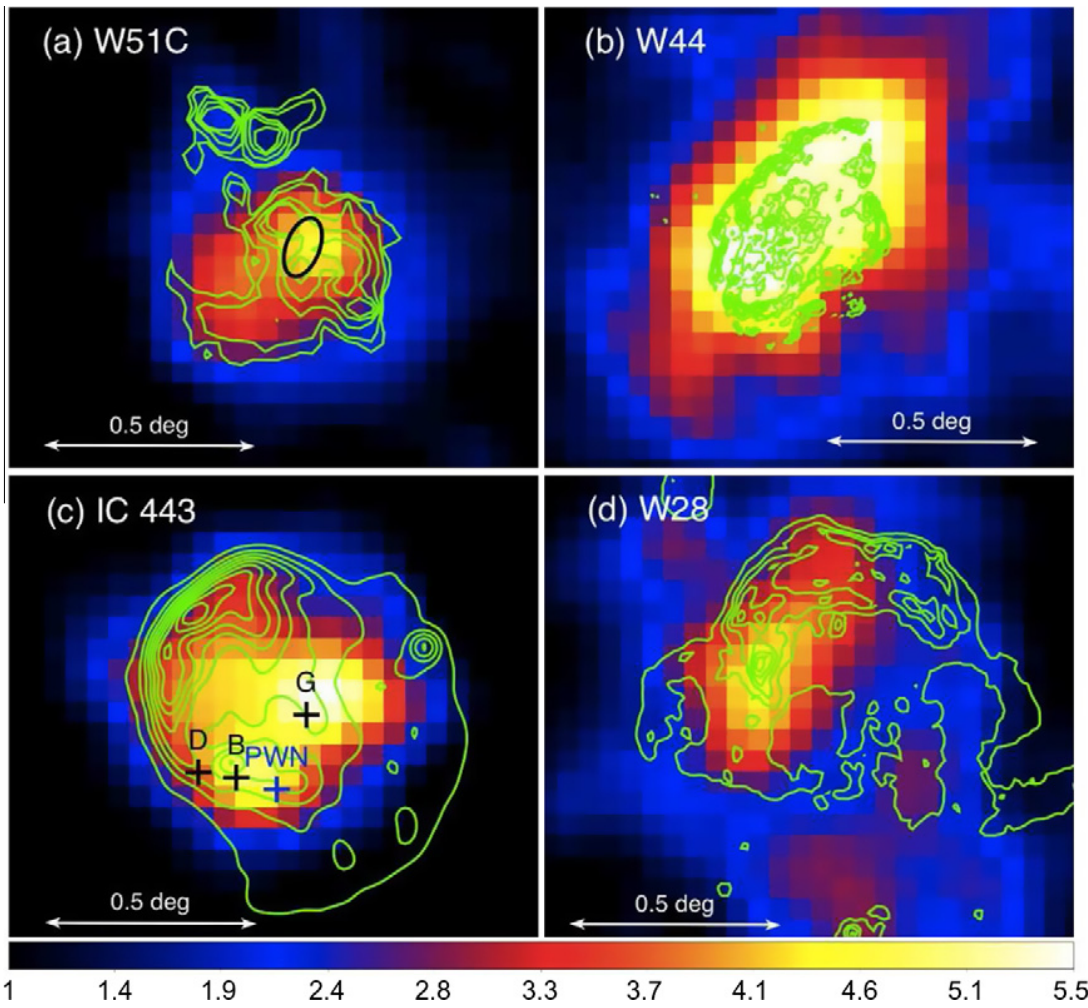

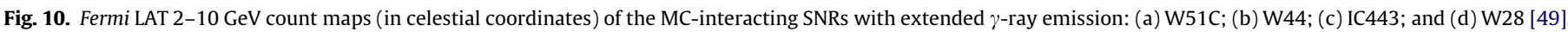

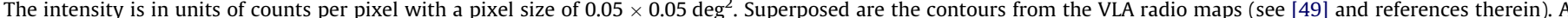

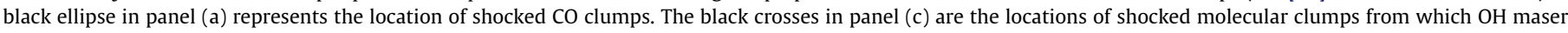
emission is detected. The position of a pulsar wind nebula is also marked in panel (c).

\subsection{SNRs interacting with molecular clouds}

The brightest $\mathrm{GeV} \gamma$-ray sources associated with SNRs are middle-aged remnants that are interacting with molecular clouds (MCs) as evidenced by the detections of $1720 \mathrm{MHz} O \mathrm{OH}$ maser emission [75]. Fig. 10 presents the raw LAT count maps in $2-10 \mathrm{GeV}$ of the four SNRs (W51C [41], W44 [42], IC 443 [43], and W28 [44]) obtained with $2.5 \mathrm{yr}$ data of the LAT observations [49]. Even before the subtraction of the intense galactic diffuse emission, the $\gamma$-ray signals from the SNRs can be easily seen.

The MC-SNRs (namely, SNRs interacting with MCs) constitute the dominant class of LAT-detected SNRs (Fig. 7). The $\gamma$-ray luminosity in the $1-10 \mathrm{GeV}$ band spans $L_{\gamma}=(0.8-9) \times 10^{35} \mathrm{erg} \mathrm{s}^{-1}$, larger than for the young SNRs like Cas A and RX J1713.7-3946 (Fig. 11). The $\mathrm{GeV} \gamma$-ray spectra commonly exhibit a spectral steepening in the Fermi LAT band, demonstrating the importance of LAT observations of these SNRs. Also, the synchrotron radio emission of the four SNRs can be characterized by a large flux density of 160$310 \mathrm{Jy}$ at $1 \mathrm{GHz}$ with flat spectral index of $\alpha \simeq 0.3-0.4$ [67]. The LAT-detected SNRs are generally radio-bright objects [49].

The predominance of the MC-SNRs among the SNRs detected by the Fermi LAT and their high $\gamma$-ray luminosity indicate that the $\gamma$ ray emission should be enhanced by the interactions with molecular clouds. Nonthermal bremsstrahlung by relativistic electrons or $\pi^{0}$-decay $\gamma$-rays produced by high-energy protons are the two plausible channels of the $\gamma$ radiation. As discussed above, the observed high luminosity of the $\mathrm{GeV} \gamma$-ray emission disfavors the IC scattering off the $\mathrm{CMB}$ and interstellar radiation fields as the emission mechanism.

There are two different types of models to account for the $\mathrm{GeV}$ $\gamma$-ray emission from the MC-SNR systems. The Runaway $C R$ model considers $\gamma$-ray emission from molecular clouds illuminated by

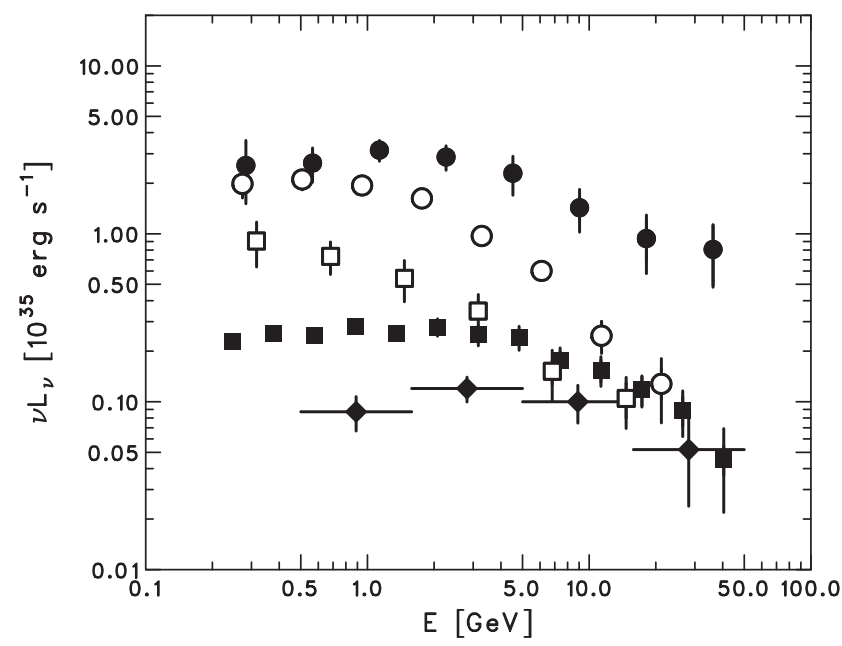

Fig. 11. Gamma-ray spectra of shell-type SNRs measured with the Fermi LAT: W51C (filled circles [41]); W44 (open circles [42]); IC 443 (filled rectangles [43]); W28 (open rectangles [44]); Cassiopeia A (filled diamonds [47]).

runaway CRs that have escaped from their accelerators, namely SNRs [76-78]. If the $\gamma$-ray emission detected by the Fermi LAT can be ascribed to the escaping CRs, one can learn about how CRs are released into interstellar space and how they propagate in the vicinity of the SNR where self-generated Alfvén waves may change the local diffusion coefficient [79]. Another scenario is the so-called Crushed Cloud model $[80,81]$ that invokes a "shocked" molecular cloud into which a radiative shock (typically $v_{s} \sim 100 \mathrm{~km} \mathrm{~s}^{-1}$ ) is driven by the impact of the SNR's blastwave. 


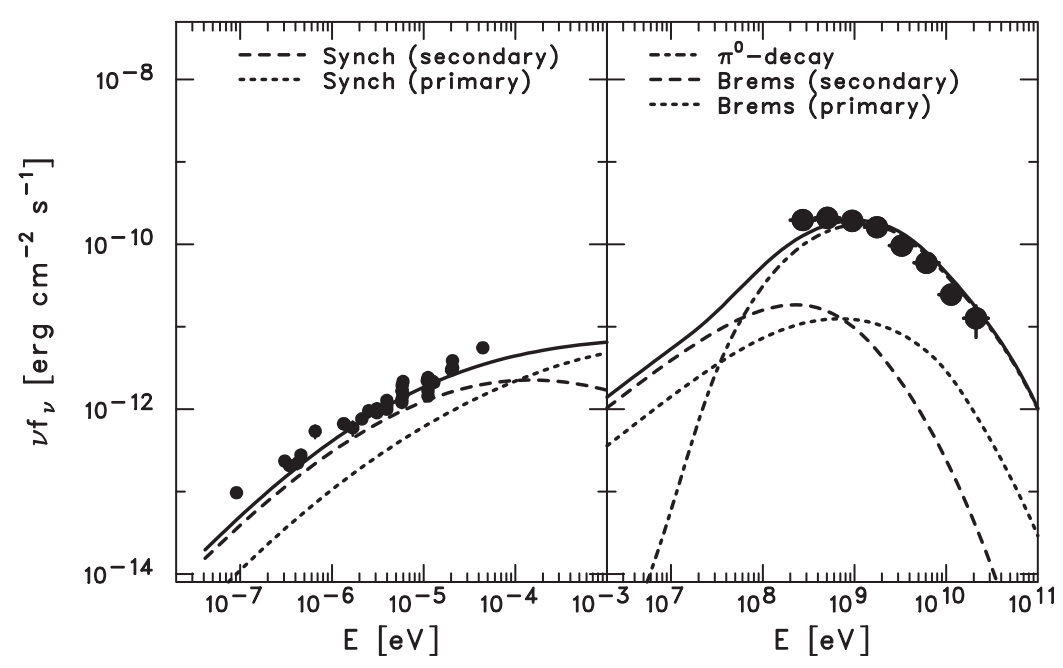

Fig. 12. Radio (left) and $\gamma$-ray (right) spectra of SNR W44 together with the reacceleration model [81].

The cosmic-ray particles accelerated at a cloud shock are adiabatically compressed behind the shock front, resulting in enhanced synchrotron and $\pi^{0}$-decay $\gamma$-ray emissions. As shown in Fig. 12 , reacceleration of pre-existing CRs at a cloud shock is capable of explaining both radio synchrotron and $\mathrm{GeV} \gamma$-ray emission in the case of SNR W44 [81]. The $\gamma$-ray luminosity of $L_{\gamma} \sim 10^{35} \mathrm{erg} \mathrm{s}^{-1}$ in 1-10 GeV agrees with the theoretical expectation. Morphologies of synchrotron radio emission support this scenario. The radio continuum map of SNR W44 exhibits filamentary and sheet-like structures of synchrotron radiation well correlated with the shocked $\mathrm{H}_{2}$ emission [82]. One can learn about shock-acceleration processes in dense environments, which complements the studies of young SNRs.

Both sites of the $\gamma$-ray emission could be important. For example, a hard $\mathrm{GeV} \gamma$-ray source [44] which is also bright in the TeV band [83] outside the southern boundary of SNR W28 might be explained by runaway CRs, while $\pi^{0}$-decay emission from radiatively-compressed layers of clouds could account for the $\mathrm{GeV} \gamma$ rays observed within SNR W28. Detailed analysis of spatial distributions of $\mathrm{GeV} \gamma$ rays combined with $\mathrm{CO}$ observations will make it possible to discern the $\gamma$-ray sources that are attributable to runaway CRs.

\subsection{Other candidate cosmic-ray sources}

Although SNR remain primary candidates for accelerating the bulk of cosmic rays, other galactic sources observed by the Fermi LAT also represent sites of particle acceleration and interaction. Such sources include pulsar wind nebulae [84], high-mass binary systems such as LS I +61 ${ }^{\circ} 303$ [85] and Cygnus X-3 [86], whose spectra seem most consistent with leptonic sources, and Nova V407 Cygni [87], a symbiotic binary system whose spectrum can be modeled with either leptonic or hadronic processes. While the energetics requirement for the $\mathrm{CR}$ protons is generally difficult to satisfy by these non-SNR sources, they might be the dominant sources of the $\mathrm{CR}$ electrons and positrons. The large number of unidentified sources found in the Fermi LAT 1FGL catalog [74] offers the possibility that there may be yet-undiscovered $C R$ production sources.

\section{Conclusions}

Both electron and $\gamma$-ray observations made with the Fermi Large Area Telescope offer new insights into cosmic-ray acceleration and interactions. The direct electron plus positron measurements suggest, although they do not prove, a possible nearby source. Future LAT observations will help measure separately the positive and negative components up to $200 \mathrm{GeV}$ and will improve on the search for anisotropies already begun. On a galactic scale, $\gamma$ rays suggest a larger scale height or flatter radial distribution of cosmic rays than previously expected. From the $\gamma$-ray studies of supernova remnants, LAT has found growing evidence that at least some SNR are likely accelerators of hadronic cosmic rays, although one example, RX J1713.7-3946, appears more consistent with being a leptonic source. The Fermi LAT continues to collect data. Improved analysis methods, coupled with the higher statistics from the ongoing mission, can confidently be expected to produce even more results addressing questions about cosmic-ray origin and propagation in the future.

\section{Acknowledgments}

The authors express their deepest thanks to the Fermi Large Area Telescope team for building this wonderful instrument and providing the opportunity for so many exciting results. We particularly thank T. Brandt, I. Moskalenko, and O. Tibolla for their valuable suggestions during the preparation of this review.

\section{References}

[1] S. Hayakawa, Propagation of the cosmic radiation through intersteller space, Prog. Theor. Phys. 8 (1952) 571-572.

[2] P. Morrison, On gamma-ray astronomy, Il Nuov. Cim. 7 (1958) 858-865

[3] W.L. Kraushaar, G.W. Clark, G.P. Garmire, R. Borken, P. Higbie, C. Leong, T Thorsos, High-energy cosmic gamma-ray observations from the OSO-3 satellite, ApJ 186 (1973) 401-402.

[4] C.E. Fichtel, R.C. Hartman, D.A. Kniffen, D.J. Thompson, H. Ögelman, M.E. Özel, T. Tumer, G.F. Bignami, High-energy gamma-ray results from the second small astronomy satellite, ApJ 198 (1975) 163-182.

[5] W. Hermsen, COS-B views on the diffuse galactic gamma-ray emission and some point sources, SSRv 49 (1989) 17-39.

[6] D.J. Thompson, Gamma ray astrophysics: the EGRET results, RPPh 71 (11) (2008) 116901.

[7] J.A. Hinton, W. Hofmann, Teraelectronvolt astronomy, ARAA 47 (2009) 523565.

[8] W.B. Atwood, A.A. Abdo, M. Ackermann, W. Althouse, B. Anderson, M. Axelsson, L. Baldini, J. Ballet, D.L. Band, G. Barbiellini, et al., The large area telescope on the Fermi gamma-ray space telescope mission, ApJ 697 (2009) 1071-1102.

[9] I.V. Moskalenko, A.W. Strong, Production and propagation of cosmic-ray positrons and electrons, ApJ 493 (1998) 694.

[10] O. Adriani, G.C. Barbarino, G.A. Bazilevskaya, et al., An anomalous positron abundance in cosmic rays with energies $1.5-100 \mathrm{GeV}$, Nature 458 (2009) 607609. 
[11] J. Chang, J.H. Adams, H.S. Ahn, et al., An excess of cosmic ray electrons at energies of 300-800 GeV, Nature 456 (2008) 362-365.

[12] A.A. Abdo, M. Ackermann, M. Ajello, et al., Measurement of the cosmic ray $e^{+}+e^{-}$spectrum from $20 \mathrm{GeV}$ to $1 \mathrm{TeV}$ with the Fermi large area telescope, PhRvL 102 (18) (2009) 181101.

[13] F. Aharonian, A.G. Akhperjanian, G. Anton, et al., Probing the ATIC peak in the cosmic-ray electron spectrum with H.E.S.S., A\&A 508 (2009) 561-564.

[14] O. Adriani, G.C. Barbarino, G.A. Bazilevskaya, et al., Cosmic-ray electron flux measured by the PAMELA experiment between 1 and $625 \mathrm{GeV}$, PhRvL 106 (20) (2011) 201101

[15] M. Ackermann, M. Ajello, W.B. Atwood, L. Baldini, et al., Fermi LAT observations of cosmic-ray electrons from $7 \mathrm{GeV}$ to $1 \mathrm{TeV}$, PhRvD 82 (9) (2010) 092004.

[16] A.W. Strong, I.V. Moskalenko, A galactic cosmic-ray database, ArXiv e-prints, <arXiv:0907.0565>

[17] D. Grasso, S. Profumo, A.W. Strong, L. Baldini, R. Bellazzini, et al., On possible interpretations of the high energy electron-positron spectrum measured by the Fermi large area telescope, APh 32 (2009) 140-151.

[18] P. Blasi, Origin of the positron excess in cosmic rays, PhRL 103 (5) (2009) 051104.

[19] B. Katz, K. Blum, J. Morag, E. Waxman, What can we really learn from positron flux anomalies?, MNRAS 405 (2010) 1458-1472

[20] F. Aharonian, A.G. Akhperjanian, U. Barres de Almeida, et al., Energy spectrum of cosmic-ray electrons at TeV energies, PhRvL 101 (26) (2008) 261104.

[21] D. Mueller, K.-K. Tang, Cosmic-ray positrons from 10 to $20 \mathrm{GeV}$ - a balloonborne measurement using the geomagnetic east-west asymmetry, ApJ 312 (1987) 183-194.

[22] International Association of Geomagnetism and Aeronomy, Working Group VMOD, International geomagnetic reference field: the eleventh generation, Geophys. J. Int. 183 (3) (2010) 1216-1230.

[23] M. Ackermann, M. Ajello, W.B. Atwood, et al., Searches for cosmic-ray electron anisotropies with the Fermi large area telescope, PhRvD 82 (9) (2010) 092003.

[24] A.W. Strong, I.V. Moskalenko, Propagation of cosmic-ray nucleons in the galaxy, ApJ 509 (1998) 212-228.

[25] A.A. Abdo, M. Ackermann, M. Ajello, B. Anderson, et al., Fermi large area telescope measurements of the diffuse gamma-ray emission at intermediate galactic latitudes, PhRvL 103 (25) (2009) 251101.

[26] A.W. Strong, I.V. Moskalenko, O. Reimer, Diffuse galactic continuum gamma rays: a model compatible with EGRET data and cosmic-ray measurements, ApJ 613 (2004) 962-976.

[27] C. Evoli, D. Gaggero, D. Grasso, L. Maccione, Cosmic ray nuclei, antiprotons and gamma rays in the galaxy: a new diffusion model, JCAP 10 (2008) 18

[28] M. Ackermann, M. Ajello, L. Baldini, et al., Constraints on the cosmic-ray density gradient beyond the solar circle from Fermi $\gamma$-ray observations of the third galactic quadrant, ApJ 726 (2011) 81.

[29] P.P. Papadopoulos, W.-F. Thi, S. Viti, Molecular gas in spiral galaxies: a new warm phase at large galactocentric distances?, ApJ 579 (2002) 270-274

[30] D. Breitschwerdt, V.A. Dogiel, H.J. Völk, The gradient of diffuse gamma-ray emission in the galaxy, A\&A 385 (2002) 216-238.

[31] G.L. Case, D. Bhattacharya, A new sigma-D relation and its application to the galactic supernova remnant distribution, ApJ 504 (1998) 761.

[32] D.A. Green, A revised galactic supernova remnant catalogue, BASI 37 (2009) 45-61.

[33] D.R. Lorimer, The galactic population and birth rate of radio pulsars, in: Fernando Camilo, Bryan M. Gaensler (Eds.), Young Neutron Stars and Their Environments, IAU Symposium No. 218, held as part of the IAU General Assembly, 14-17 July, 2003 in Sydney, Australia. . San Francisco, CA: Astronomical Society of the Pacific, 2004, p. 105.

[34] A.A. Abdo, M. Ackermann, M. Ajello, L. Baldini, et al., Detection of the small magellanic cloud in gamma-rays with Fermi/LAT, A\&A 523 (2010) A46.

[35] A.A. Abdo, M. Ackermann, M. Ajello, W.B. Atwood, et al., Observations of the large magellanic cloud with Fermi, A\&A 512 (2010) A7.

[36] A.A. Abdo, M. Ackermann, M. Ajello, W.B. Atwood, et al., Detection of gammaray emission from the starburst galaxies M82 and NGC 253 with the large area telescope on Fermi, ApJ 709 (2010) L152-L157.

[37] V.L. Ginzburg, S.I. Syrovatskii, The Origin of Cosmic Rays, Macmillan, New York, 1964.

[38] R. Blandford, D. Eichler, Particle acceleration at astrophysical shocks: a theory of cosmic ray origin, Phys. Rep. 154 (1987) 1-75.

[39] A.M. Hillas, Topical review: can diffusive shock acceleration in supernova remnants account for high-energy galactic cosmic rays?, JPhGNP 31 (2005) 95

[40] L.O. Drury, F.A. Aharonian, H.J. Voelk, The gamma-ray visibility of supernova remnants. A test of cosmic ray origin, A\&A 287 (1994) 959-971.

[41] A.A. Abdo, M. Ackermann, M. Ajello, L. Baldini, et al., Fermi LAT discovery of extended gamma-ray emission in the direction of supernova remnant W51C ApJ 706 (2009) L1-L6.

[42] A.A. Abdo, M. Ackermann, M. Ajello, L. Baldini, et al., Gamma-ray emission from the shell of supernova remnant W44 revealed by the Fermi LAT, Science 327 (2010) 1103

[43] A.A. Abdo, M. Ackermann, M. Ajello, L. Baldini, et al., Observation of supernova remnant IC 443 with the Fermi large area telescope, ApJ 712 (2010) 459-468.

[44] A.A. Abdo, M. Ackermann, M. Ajello, A. Allafort, et al., Fermi large area telescope observations of the supernova remnant W28 (G6.4-0.1), ApJ 718 (2010) 348-356.
45] A.A. Abdo, M. Ackermann, M. Ajello, L. Baldini, J. Ballet, et al., Fermi-LAT study of gamma-ray emission in the direction of supernova remnant W49B, ApJ 722 (2010) 1303-1311.

[46] D. Castro, P. Slane, Fermi large area telescope observations of supernova remnants interacting with molecular clouds, ApJ 717 (2010) 372-378.

[47] A.A. Abdo, M. Ackermann, M. Ajello, A. Allafort, et al., Fermi-lat discovery of $\mathrm{GeV}$ gamma-ray emission from the young supernova remnant CassiopeiaA ApJ 710 (2010) L92-L97.

[48] A.A. Abdo, M. Ackermann, M. Ajello, A. Allafort, L. Baldini, et al., Observations of the young supernova remnant RX J1713.7-3946 with the Fermi large area telescope, ApJ 734 (2011) 28.

49] Y. Uchiyama, on behalf of the Fermi LAT collaboration, GeV gamma rays from supernova remnants interacting with molecular clouds, Proceedings of Science, 2011. <arXiv:1104.1197>.

[50] A.R. Bell, Turbulent amplification of magnetic field and diffusive shock acceleration of cosmic rays, MNRAS 353 (2004) 550-558.

[51] J. Vink, J.M. Laming, On the magnetic fields and particle acceleration in Cassiopeia A, ApJ 584 (2003) 758-769.

[52] A. Bamba, R. Yamazaki, M. Ueno, K. Koyama, Small-scale structure of the SN 1006 shock with Chandra observations, ApJ 589 (2003) 827-837.

[53] Y. Uchiyama, F.A. Aharonian, T. Tanaka, T. Takahashi, Y. Maeda, Extremely fast acceleration of cosmic rays in a supernova remnant, Nature 449 (2007) 576578.

[54] V.S. Ptuskin, F.C. Jones, E.S. Seo, R. Sina, Effect of random nature of cosmic ray sources supernova remnants on cosmic ray intensity fluctuations, anisotropy, and electron energy spectrum, AdSR 37 (2006) 1909-1912.

[55] P. Blasi, E. Amato, Diffusive propagation of cosmic rays from supernova remnants in the Galaxy. II: anisotropy, ArXiv e-prints, 2011.<arXiv:1105.4529>.

[56] D. Caprioli, E. Amato, P. Blasi, The contribution of supernova remnants to the galactic cosmic ray spectrum, APh 33 (2010) 160-168.

[57] F. Aharonian, A. Akhperjanian, J. Barrio, K. Bernlöhr, H. Börst, et al., Evidence for TeV gamma ray emission from Cassiopeia A, A\&A 370 (2001) 112-120.

[58] E. Parizot, A. Marcowith, J. Ballet, Y.A. Gallant, Observational constraints on energetic particle diffusion in young supernovae remnants: amplified magnetic field and maximum energy, A\&A 453 (2006) 387-395.

[59] Y. Uchiyama, F.A. Aharonian, Fast variability of nonthermal x-ray emission in Cassiopeia A: probing electron acceleration in reverse-shocked ejecta, ApJ 677 (2008) L105-L108

[60] J. Albert, E. Aliu, H. Anderhub, P. Antoranz, A. Armada, et al., Observation of VHE $\gamma$-rays from Cassiopeia A with the MAGIC telescope, A\&A 474 (2007) 937940

[61] T.B. Humensky, VERITAS studies of the supernova remnants Cas A and IC 443, in: F.A. Aharonian, W. Hofmann, F. Rieger (Eds.), American Institute of Physics Conference Series.

[62] F. Aharonian, A.G. Akhperjanian, A.R. Bazer-Bachi, M. Beilicke, W. Benbow et al., Primary particle acceleration above $100 \mathrm{TeV}$ in the shell-type supernova remnant RX J1713.7-3946 with deep H.E.S.S. observations, A\&A 464 (2007) 235-243.

[63] V.A. Acciari, E. Aliu, T. Arlen, et al., Discovery of TeV gamma-ray emission from Tycho's supernova remnant, ApJL 730 (2011) L20.

[64] F. Giordano, M. Naumann-Godo, J. Ballet, K. Bechtol, S. Funk, J. Lande, M.N.Mazziotta, S. Rainò, T. Tanaka, O. Tibolla, Y. Uchiyama, Fermi-LAT detection of the young supernova remnant Tycho, ArXiv e-prints, 2011. <arXiv:1108.0265>

[65] H.J. Völk, E.G. Berezhko, L.T. Ksenofontov, Magnetic field amplification in Tycho and other shell-type supernova remnants, A\&A 433 (2005) 229-240.

[66] G. Morlino, D. Caprioli, The unequivocal evidence of hadron acceleration in Tycho's supernova remnant, ArXiv e-prints, <arXiv:1105.6342>.

[67] D.A. Green, A Catalogue of Galactic Supernova Remnants (Green 2009), VizieR Online Data Catalog, Originally published in 2009, BASI, 37, 457253 (2009) 0

[68] K. Koyama, K. Kinugasa, K. Matsuzaki, M. Nishiuchi, M. Sugizaki, K. Torii, S. Yamauchi, B. Aschenbach, Discovery of non-thermal X-rays from the northwest shell N3.7-3946: the second SN 1006?, PASJ 49 (1997) L7-L11

[69] P. Slane, B.M. Gaensler, T.M. Dame, J.P. Hughes, P.P. Plucinsky, A. Green, Nonthermal X-ray emission from the shell-type supernova remnant G347.3 0.5 , ApJ 525 (1999) 357-367.

[70] F.A. Aharonian, A.G. Akhperjanian, K.-M. Aye, A.R. Bazer-Bachi, M. Beilicke et al., High-energy particle acceleration in the shell of a supernova remnant, Nature 432 (2004) 75-77.

[71] V.N. Zirakashvili, F.A. Aharonian, Nonthermal radiation of young supernova remnants: the case of RX J1713.7-3946, ApJ 708 (2010) 965-980.

[72] D.C. Ellison, D.J. Patnaude, P. Slane, J. Raymond, Efficient cosmic ray acceleration, hydrodynamics, and self-consistent thermal X-ray emission applied to supernova remnant RX J1713.7-3946, ApJ 712 (2010) 287-293.

[73] A.M. Bykov, Y.A. Uvarov, J.B.G.M. Bloemen, J.W. den Herder, J.S. Kaastra, A model of polarized X-ray emission from twinkling synchrotron supernova shells, MNRAS 399 (2009) 1119-1125.

[74] A.A. Abdo, M. Ackermann, M. Ajello, A. Allafort, E. Antolini, W.B. Atwood, M. Axelsson, L. Baldini, J. Ballet, G. Barbiellini, et al., Fermi large area telescope first source catalog, ApJS 188 (2010) 405-436.

[75] J.W. Hewitt, F. Yusef-Zadeh, M. Wardle, Correlation of supernova remnant masers and gamma-ray sources, ApJL 706 (2009) L270-L274.

[76] F.A. Aharonian, A.M. Atoyan, On the emissivity of $\pi^{0}$-decay gamma radiation in the vicinity of accelerators of galactic cosmic rays, A\&A 309 (1996) 917928. 
[77] S. Gabici, F.A. Aharonian, S. Casanova, Broad-band non-thermal emission from molecular clouds illuminated by cosmic rays from nearby supernova remnants, MNRAS 396 (2009) 1629-1639.

[78] Y. Ohira, K. Murase, R. Yamazaki, Gamma-rays from molecular clouds illuminated by cosmic rays escaping from interacting supernova remnants, MNRAS 410 (2011) 1577-1582.

[79] Y. Fujita, Y. Ohira, F. Takahara, Slow diffusion of cosmic rays around a supernova remnant, ApJ 712 (2010) L153-L156.

[80] R.D. Blandford, L.L. Cowie, Radio emission from supernova remnants in a cloudy interstellar medium, ApJ 260 (1982) 625-634.

[81] Y. Uchiyama, R.D. Blandford, S. Funk, H. Tajima, T. Tanaka, Gamma-ray emission from crushed clouds in supernova remnants, ApJ 723 (2010) L122L126.

[82] W.T. Reach, J. Rho, T.H. Jarrett, Shocked molecular gas in the supernova remnants W28 and W44: near-infrared and millimeter-wave observations, Ap] 618 (2005) 297-320.
[83] F. Aharonian, A.G. Akhperjanian, A.R. Bazer-Bachi, B. Behera, M. Beilicke, et al., A\&A 481 (2008) 401-410.

[84] M. Ackermann, M. Ajello, L. Baldini, J. Ballet, et al., Fermi-LAT search for pulsar wind nebulae around gamma-ray pulsars, ApJ 726 (2011) 35.

[85] A.A. Abdo, M. Ackermann, M. Ajello, W.B. Atwood, et al., Fermi LAT observations of LS I+61303: first detection of an orbital modulation in $\mathrm{GeV}$ gamma rays, ApJ 701 (2009) L123-L128.

[86] A.A. Abdo, M. Ackermann, M. Ajello, M. Axelsson, et al., Fermi LAT Collaboration, Modulated high-energy gamma-ray emission from the microquasar Cygnus X-3, Science 326 (2009) 1512.

[87] A.A. Abdo, M. Ackermann, M. Ajello, W.B. Atwood, L. Baldini, J. Ballet, G. Barbiellini, D. Bastieri, K. Bechtol, R. Bellazzini, et al., Gamma-ray emission concurrent with the nova in the symbiotic binary V407 Cygni, Science 329 (2010) 817-821 